\title{
Ocorrência de Plantas Aquáticas nos Reservatórios da Companhia Energética de São Paulo ${ }^{1}$
}

\author{
Aquatic Weed Survey in Reservoirs Controlled by the Power Plant of São Paulo, Brazil
}

TANAKA, R.H. ${ }^{2}$, CARDOSO, L.R. ${ }^{3}$, MARTINS, D. ${ }^{4}$, MARCONDES, D.A.S. ${ }^{5}$ e MUSTAFÁ, A.L. ${ }^{6}$

\begin{abstract}
RESUMO - O objetivo do presente trabalho foi caracterizar as comunidades de plantas aquáticas presentes nos reservatórios da Companhia Energética de São Paulo. Os levantamentos foram realizados entre janeiro e dezembro de 1999, percorrendo-se com um barco as margens dos reservatórios de Três Irmãos, Jupiá, Ilha Solteira, Porto Primavera, Paraibuna e Jaguari, visando identificar as áreas com infestações de plantas aquáticas. Em cada ponto de avaliação, fez-se a identificação das espécies e estimou-se visualmente a área total infestada e a participação de cada espécie (em \% da área total). Com as informações coletadas em campo, procedeu-se a uma etapa de trabalhos em escritório, incluindo a identificação das espécies de plantas aquáticas nos casos em que não era possível identificar as espécies no local; a determinação de classes de plantas aquáticas (emersas, emersas com folhas flutuantes, submersas, flutuantes); a identificação das espécies mais freqüentes; e o estabelecimento de relações de dominância e co-dominância. São apresentados os resultados obtidos em cada reservatório.
\end{abstract}

Palavras-chave: planta aquática, levantamento, CESP.

\begin{abstract}
This work aimed to describe the aquatic plant communities present in reservoirs controlled by Companhia Energética de São Paulo (CESP). The survey was carried out from January to December 1999, using a boat along the marginal areas of Três Irmãos, Jupiá, Ilha Solteira, Porto Primavera, Paraibuna and Jaguari reservoirs, aiming to identify areas with aquatic plant infestations. In each evaluation point plant species were identified and the infested areas and participation of each species visually estimated (\% of the total area). Fieldwork for data collection were followed by other studies including the identification of plant species not identified in the field; the classification of plants in groups (emersed, emersed with floating leaves, submersed and floating); the identification of the most frequent species and the determination of dominance and co-dominance relations. Results are presented for each reservoir.
\end{abstract}

Key words: aquatic plant, survey, CESP.

\section{INTRODUÇÃO}

As plantas aquáticas são componentes importantes em corpos hídricos, desempenhando papel fundamental na manutenção do equilíbrio dos ecossistema aquáticos. No entanto, em certas condições, elas podem se tornar um problema, devido ao seu rápido crescimento. Como as plantas aquáticas têm grande influência sobre os processos físico-químicos de um ecossistema e, simultaneamente, afetam atividades humanas, é essencial que seu desenvolvimento seja vistoriado continuamente, de forma a permitir a adoção de práticas de manejo, caso haja necessidade.

Recebido para publicação em 4/1/2002 e na forma revisada em 7/2/2002.

2 Eng.-Agr., M.S., Companhia Energética de São Paulo, Rua da Consolação, 1875, 01301-100 São Paulo-SP. ${ }^{3}$ Eng.-Agr., M.S., Dep. de Produção Vegetal, FCA-UNESP, Caixa Postal 237, 18603-970 Botucatu-SP; ${ }^{4}$ Prof. Assistente, Dr., Dep. de Produção Vegetal, FCA-UNESP. ${ }^{5}$ Eng.-Agr., Dr., Companhia Energética de São Paulo, Rua da Consolação, 1875, 01301-100 São PauloSP; ${ }^{6}$ Eng.-Agr., Companhia Energética de São Paulo. 
O levantamento periódico da ocorrência de plantas aquáticas permite avaliar a evolução das comunidades e, respaldado por outras atividades, como os estudos de qualidade de água, possibilita analisar as causas de eventuais alterações. Essa atividade tem duas funções básicas: permitir o acompanhamento de tendências nas comunidades de plantas aquáticas ou flutuações populacionais de uma planta ou grupo de plantas; e proporcionar informações confiáveis para o estabelecimento de prioridades do manejo de plantas aquáticas (Schardt, 1992).

A identificação das espécies de plantas aquáticas em um corpo de água é uma etapa crítica em planos de manejo, por várias razões. Primeiro, espécies diferentes freqüentemente respondem de forma diferente às técnicas de controle. Também é importante determinar se alguma planta rara ou de importância fundamental no ecossistema está presente. Finalmente, é crucial descobrir se qualquer planta exótica ou alóctone está presente (Schardt \& Ludlow, 1993).

Apesar de ter inegável importância, o levantamento da ocorrência de plantas aquáticas ainda é um grande desafio, pois elas são de grupos bastante distintos (emersas, flutuantes, submersas) e ocorrem em ampla variação de ambientes (de áreas de várzeas até profundidades superiores a 10 metros), além de haver enormes variações de espécies e distribuição espacial de um ano para outro. Por esse motivo, há grande quantidade de metodologias de avaliação de infestações de plantas aquáticas, podendo ser atividades simples, envolvendo apenas a identificação das espécies em campo, até o uso de imagens de satélite ou fotos aéreas, com quantificação das áreas ocupadas por cada espécie ou grupo (Malthus \& George, 1997; Jensen \& Davis, 1987).

Este projeto teve por objetivo levantar a ocorrência de plantas aquáticas nos reservatórios de usinas hidrelétricas da Companhia Energética de São Paulo (CESP).

\section{MATERIAL E MÉTODOS}

Foram consideradas aquáticas as plantas vasculares, visíveis a olho nu, que se desenvolvem em ambientes alagados (Pedralli, 1990). As plantas aquáticas foram classificadas em quatro grupos:
- Emersas: são enraizadas e apresentam a maior parte do caule e das folhas acima da superfície da água, desenvolvendo-se em locais rasos ou ao longo da margem.

- Emersas com folhas flutuantes: são enraizadas e têm as folhas flutuantes, conectadas por caules submersos.

- Submersas: possuem a maior parte das folhas e do caule abaixo da superfície da água, muitas vezes com flores acima desta superfície; podem ser enraizadas ou livres.

- Flutuantes: suas raízes ficam livres na água e apresentam folhas e flores acima da superfície da água.

A atividade foi dividida em duas etapas: coleta de dados no reservatório e tabulação e análise dos dados.

\section{Coleta de dados no reservatório}

Nesta etapa, desenvolvida entre janeiro e dezembro de 1999, percorreu-se as margens dos seis reservatórios operados pela Companhia Energética de São Paulo (Três Irmãos, Jupiá, Ilha Solteira, Porto Primavera, Paraibuna e Jaguari - Figura 1) com um barco, visando identificar as áreas com infestações de plantas aquáticas. As áreas com plantas aquáticas foram fotografadas e identificadas com um aparelho localizador geográfico Garmin GPS 12.

Como o objetivo deste trabalho foi fazer uma amostragem preliminar do tipo de infestação do reservatório, utilizou-se a seguinte metodologia para demarcação dos pontos de avaliação: a cada 15 minutos navegados, fez-se a leitura das coordenadas no localizador geográfico e um gancho foi arrastado no fundo do reservatório para verificar a presença ou ausência de plantas submersas. Nos pontos com plantas emersas e submersas atingindo a superfície, fez-se a identificação das espécies e a avaliação da área total infestada e da composição da participação de cada espécie (em \% da área total). Nos pontos em que as plantas submersas não atingiam a superfície da água, não se fez a avaliação da área infestada, sendo registrada apenas a presença da espécie. Nos casos em que não era possível identificar a espécie, uma amostra foi coletada e preservada em sacos plásticos com água, para posterior identificação pela UNESP. Além desses pontos, outros foram 
definidos, de acordo com o interesse, podendo ser: entradas de tributários, infestações encontradas ao longo do rio (entre os pontos, a cada 20 minutos) ou pontos definidos previamente.

\section{Tabulação e análise dos dados}

Com as informações coletadas em campo, procedeu-se a uma etapa de trabalhos em escritório, incluindo:

- Identificação das espécies de plantas aquáticas: nos casos em que não era possível identificar as espécies durante a coleta de dados, uma amostra das plantas era coletada e encaminhada à UNESP. Em muitos casos foi possível descobrir a espécie, mas, em outros, chegava-se apenas ao gênero. Nestes casos, as plantas foram classificadas apenas pelas três primeiras letras do código internacional, como, por exemplo, POL, de Polygonum spp. Ressalta-se que a dificuldade de identificação correta das espécies deveu-se principalmente à pequena quantidade de referências bibliográficas nacionais.

- Determinação de classes de plantas aquáticas (emersas, emersas com folhas flutuantes, submersas, flutuantes).
- Determinação do nível de infestação nos pontos de monitorização: as áreas ocupadas por plantas aquáticas foram agrupadas em cinco níveis, de acordo com sua dimensão (Tabela 1).

- Identificação das espécies mais freqüentes.

- Identificação de relações de dominância, codominância: de acordo com Schardt (1992), uma espécie era definida como dominante quando cobria mais de $50 \%$ da área avaliada, sendo a avaliação realizada visualmente ou arrastando-se rapidamente um gancho no fundo do rio por três vezes. Nos casos em que não se obtinham amostras de plantas, amostragens mais longas (arrastando-se o gancho por mais tempo) ou um número maior de amostragens eram utilizados para verificar se realmente não havia plantas presentes. Duas espécies eram classificadas como co-dominantes se elas estivessem misturadas e tivessem uma cobertura combinada superior a $50 \%$ da área avaliada. $\mathrm{Se}$ as duas espécies mais comuns ocupassem menos de $50 \%$ da área avaliada, definia-se não haver espécie dominante.

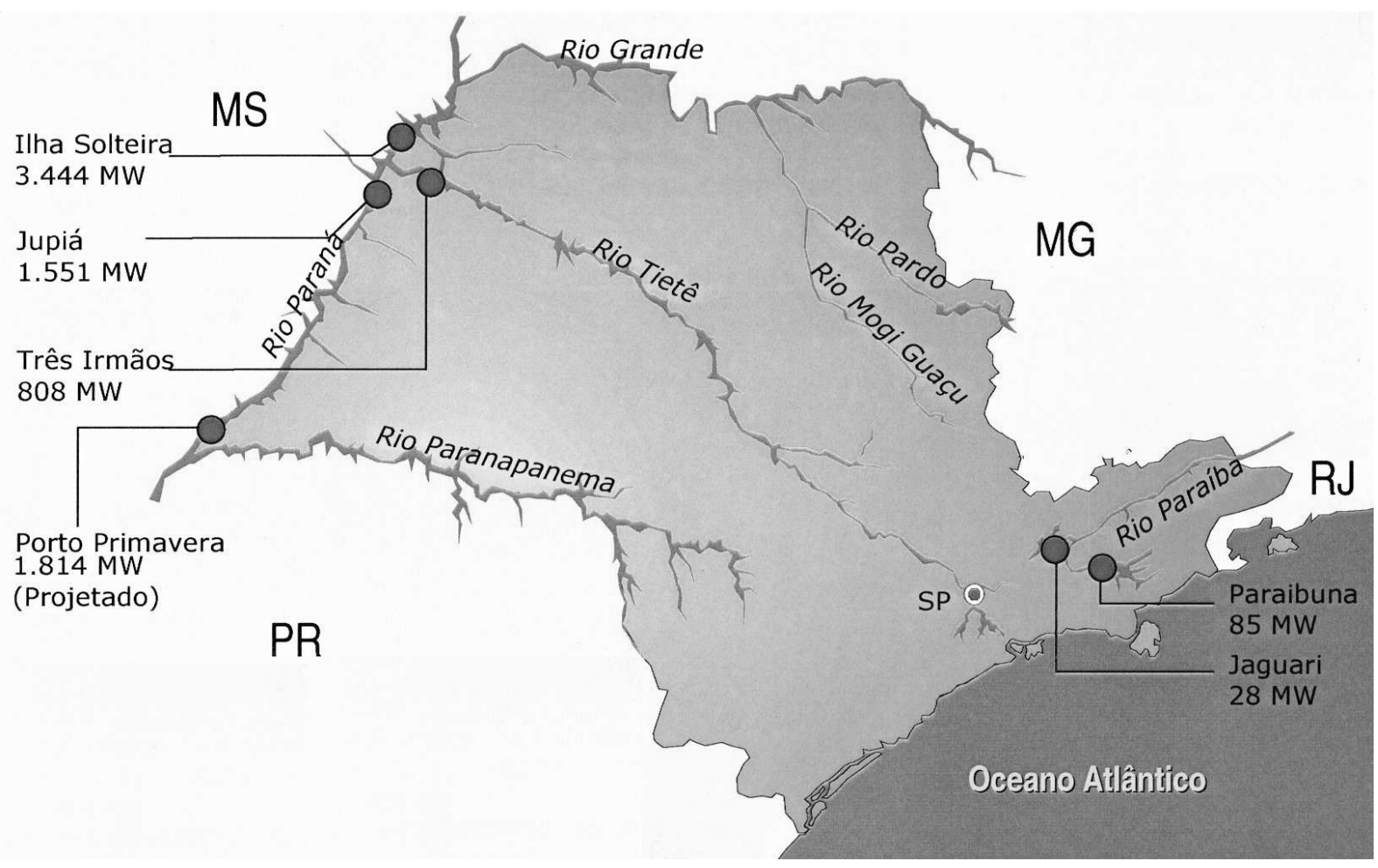

Figura 1 - Localização das usinas da CESP. 
Tabela 1 - Classes de níveis de infestação

\begin{tabular}{|c|c|}
\hline Nível de infestação & Área $\left(\mathrm{m}^{2}\right)$ \\
\hline 1 & 0 \\
2 & $1-100$ \\
3 & $101-1.000$ \\
4 & $1.001-10.000$ \\
5 & $>10.000$ \\
\hline
\end{tabular}

\section{RESULTADOS E DISCUSSÃO}

Nos levantamentos realizados nos seis reservatórios, foram identificadas 29 espécies de plantas aquáticas, apresentadas na Tabela 2. As espécies identificadas representam 17 famílias diferentes.

De acordo com a metodologia descrita anteriormente, foram elaboradas as Tabela 3 a 8, com a avaliação da área ocupada pelas plantas aquáticas em cada reservatório, o nível de infestação e a composição da população em cada ponto de avaliação. Também são apresentadas as coordenadas destes pontos, o que permite a construção de mapas de infestação de plantas aquáticas em cada reservatório. A Tabela 9 apresenta um resumo dos dados das Tabelas 3 a 8.

Tabela 2 - Espécies de plantas aquáticas identificadas nos reservatórios da CESP

\begin{tabular}{|c|c|c|c|c|}
\hline Nome científico & Família & Nomes vulgares & Código & Grupo \\
\hline Azolla filiculoides Lam. & Azollaceae & azola, samambaia-aquática & AZOFI & FL \\
\hline Brachiaria arrecta (Hack.) Stent. & Poaceae & tanner-grass & BRASU & EM \\
\hline Brachiaria mutica (Forsk.) Stapf & Poaceae & capim-de-angola, capim-fino & PANPU & $\mathrm{EM}$ \\
\hline Ceratophyllum demersum L. & "Cetatophyllaceae & Candelabro-aquático & CERDE & $\mathrm{SU}$ \\
\hline Commelina spp. & Commelinaceae & trapoeraba & $\mathrm{COM}$ & EM \\
\hline Cyperus spp. & Cyperaceae & lunquinho, tiririca & CYP & $\mathrm{EM}$ \\
\hline Echinochloa polystachya (Kunth) Hitch. & Poaceae & ' canarana, capim-capivara & ECHPO & EM \\
\hline Egeria densa Planch. & Hydrocharitaceae & egeria, elódea-brasileira & EGRDE & $\mathrm{SU}$ \\
\hline Egeria najas Planch. & "Hydrocharitaceae & lodinho & EGRNA & $\mathrm{SU}$ \\
\hline Eichhornia azurea (Sw.) Kunth & Pontederiaceae & aguapé-de-cordão & EICAZ & $\mathrm{EM}$ \\
\hline Eichhornia crassipes (Mart.) Solms & Pontederiaceae & aguapé, aguapé-de-flor-roxa & EICCR & FL \\
\hline Eleocharis spp. & Cyperaceae & junco-manso & ELO & EM \\
\hline Hydrocotyle umbellata L. & Umbelliferae & pára-sol, erva-capitão, cicuta-falsa & HYDUM & EM \\
\hline Limnobium spongia & Hydrocharitaceae & & LIMSP & $\mathrm{FF} / \mathrm{EM}$ \\
\hline Ludwigia spp. & Onagraceae & cruz-de-malta & LUD & $\mathrm{EM}$ \\
\hline Myriophyllum brasiliense (Vell.) Verdc. & Haloragaceae & pinheirinho-d'água, bem-casados & MYPBR & SU \\
\hline Najas guadalupensis & Najadaceae & & NAJGU & $\mathrm{SU}$ \\
\hline Nymphaea spp. & Nymphaceae & írio-aquático & NYM & $\mathrm{FF}$ \\
\hline Nymphaea ampla DC. & Nymphaceae & aguapé-da-flor-grande & NYMAM & FF \\
\hline Paspalum repens Berg. & Poaceae & canarana-rasteira & PASRE & $\mathrm{EM}$ \\
\hline Pistia stratiotes L. & Araceae & alface-d’água & PISST & FL \\
\hline Polygonum spp. & Polygonaceae & erva-de-bicho & POL & EM \\
\hline Polygonum hydropiperoides Michaux & Polygonaceae & erva-de-bicho, cataia & POLHP & EM \\
\hline Polygonum lapathifolium L. & Polygonaceae & erva-de-bicho, cataia & POLLA & $\mathrm{EM}$ \\
\hline Polygonum plathyphylla. & Polygonaceae & erva-de-bicho & POLPL & EM \\
\hline Salvinia minima Bak. & Salviniaceae & salvínia, samambaia-aquática & SAVMI & FL \\
\hline Salvinia molesta & Salviniaceae & salvínia, samambaia-aquática & SAVMO & FL \\
\hline Typha latofolia Forst. & Typhaceae & taboa & TYPLA & $\mathrm{EM}$ \\
\hline Utricularia gibba L. & Lentibulariaceae & mururé & UTRGI & $\mathrm{SU}$ \\
\hline
\end{tabular}

Grupo EM - emersa, FF - emersa com folhas flutuantes, FL - flutuante e SU - submersa. 
Tabela 3 - Composição da comunidade nos pontos de avaliação do reservatório de Ilha Solteira

\begin{tabular}{|c|c|c|c|c|c|c|c|c|c|c|c|c|c|c|c|c|c|c|}
\hline \multirow{2}{*}{$\begin{array}{l}\text { Ponto de } \\
\text { avaliaçãoo }\end{array}$} & \multirow{2}{*}{\multicolumn{2}{|c|}{$\begin{array}{c}\text { Coordenadas } \\
\text { UTM }\end{array}$}} & \multirow{2}{*}{$\begin{array}{l}\text { Área } \\
\left(\mathrm{m}^{2}\right)\end{array}$} & \multirow{2}{*}{$\begin{array}{c}\text { Nível de } \\
\text { infes- } \\
\text { tação }\end{array}$} & \multicolumn{14}{|c|}{ Infestação de plantas aquáticas (\%) } \\
\hline & & & & & EGRNJ & EICCR & EICAZ & POL & SAVMO & ELO & CYP & POLPL & POLHP & PANPU & PISST & ECHPO & NYM & NYMAM \\
\hline 1 & 563769 & 7807068 & 100 & 2 & & & 100 & & & & & & & & & & & \\
\hline 2 & 563087 & 7808080 & 250 & 3 & & 30 & 40 & & 1 & & 9 & 20 & & & & & & \\
\hline 3 & 562533 & 7808577 & 100 & 2 & 100 & & & & & & & & & & & & & \\
\hline 4 & 562673 & 7808567 & 10.000 & 4 & 65 & 5 & 15 & & 2 & & & 10 & 3 & & & & & \\
\hline 5 & 558274 & 7810733 & 1.000 & 3 & 5 & 30 & & & 5 & & 20 & 30 & & & & 10 & & \\
\hline 6 & 555945 & 7810444 & 7.000 & 4 & & 60 & & & 4 & & 5 & 30 & & & 1 & & & \\
\hline 7 & 553083 & 7811405 & & 1 & & & & & & & & & & & & & & \\
\hline 8 & 549517 & 7810163 & & 1 & & & & & & & & & & & & & & \\
\hline 9 & $548914 \mid$ & 7809440 & 8.000 & 4 & 90 & & 5 & & & & & & 5 & & & & & \\
\hline 10 & 548866 & 7809261 & 600 & 3 & 5 & 30 & & & 5 & & 10 & & & 50 & & & & \\
\hline 11 & $547717 \mid$ & 7809811 & 200 & 3 & 0 & 60 & & & & & & 40 & & & & & & \\
\hline 12 & 547432 & 7809909 & 1.000 & 3 & & 40 & & & 5 & & 5 & & 50 & & & & & \\
\hline 13 & $548211 \mid$ & 7809952 & 0 & 2 & 100 & & & & & & & & & & & & & \\
\hline 14 & $|551020|$ & 7811618 & & 1 & & & & & & & & & & & & & & \\
\hline 15 & 556516 & 7813579 & & 1 & & & & & & & & & & & & & & \\
\hline 16 & $558604 \mid$ & 7810995 & 2.000 & 4 & & 1 & & & & & & & & 95 & 4 & & & \\
\hline 17 & 560189 & 7810951 & 500 & 3 & 100 & & & & & & & & & & & & & \\
\hline 18 & 561235 & 7810181 & 300 & 3 & 10 & 10 & & & & & & 80 & & & & & & \\
\hline 19 & 562966 & 7808444 & 1.500 & 4 & 5 & 5 & & & 2 & & 13 & & 30 & 45 & & & & \\
\hline 20 & 563526 & 7808551 & 2.000 & 4 & 20 & 20 & & & & & 35 & 25 & & & & & & \\
\hline 21 & 565374 & 7806038 & 200 & 3 & 10 & 5 & & & & & 80 & & & & & & & 5 \\
\hline 22 & $566376 \mid$ & 7804419 & 2.000 & 4 & 100 & & & & & & & & & & & & & \\
\hline 23 & 493155 & 7725901 & 500 & 3 & 30 & & 40 & 15 & & 10 & & & & & & & 5 & \\
\hline 24 & 493813 & 7726588 & 800 & 3 & 30 & & 25 & 15 & & 30 & & & & & & & & \\
\hline 25 & 497363 & 7725172 & $\begin{array}{l}300 \\
300\end{array}$ & 3 & 20 & 5 & 25 & 10 & 5 & 30 & & & & & 5 & & & \\
\hline 26 & 499636 & 7726980 & 500 & 3 & 10 & & 10 & 25 & 5 & 50 & & & & & & & & \\
\hline 27 & $|494824|$ & 7728769 & 1.500 & 4 & 30 & & 40 & 15 & & 15 & & & & & & & & \\
\hline 28 & 488239 & 7729954 & 15.000 & 5 & 50 & & 5 & 40 & & 5 & & & & & & & & \\
\hline 29 & 485578 & 7729737 & 1.500 & 4 & 50 & & 15 & 30 & & 5 & & & & & & & & \\
\hline 30 & 484197 & 7733644 & 2.000 & 4 & 50 & & 10 & 30 & 5 & 5 & & & & & & & & \\
\hline 31 & 486912 & 7729836 & 5.000 & 4 & 60 & 5 & 10 & 10 & & 15 & & & & & & & & \\
\hline 32 & 486810 & 7723471 & 2.000 & 4 & 10 & 20 & & 20 & & 50 & & & & & & & & \\
\hline \multicolumn{4}{|c|}{$\mathrm{N}^{\mathbf{0}}$ de pontos com plantas da espécie } & & 23 & 15 & 13 & 10 & 10 & 10 & 8 & 7 & 4 & 3 & 3 & 1 & 1 & 1 \\
\hline \multicolumn{4}{|c|}{$\mathrm{N}^{\mathrm{o}}$ de pontos com dominância } & & 10 & 2 & 1 & 0 & 0 & 2 & 1 & 1 & 1 & 2 & 0 & 0 & 0 & 0 \\
\hline \multicolumn{4}{|c|}{$\mathrm{N}^{\mathrm{o}}$ de pontos com co-dominância } & & 3 & 2 & 4 & 0 & 0 & 2 & 1 & 2 & 1 & 1 & 0 & 0 & 0 & 0 \\
\hline
\end{tabular}

Legenda: Nível de infestação: $1=0 \mathrm{~m}^{2} ; 2=1$ a $100 \mathrm{~m}^{2} ; 3=101$ a $1.000 \mathrm{~m}^{2} ; 4=1.001$ a $10.000 \mathrm{~m}^{2}$; e $5=>10.000 \mathrm{~m}^{2}$.

Espécie dominante: ocupa $50 \%$ ou mais da área infestada. Espécies co-dominantes: duas espécies com cobertura combinada de 50\% ou mais da área infestada. 
Tabela 4 - Composição da comunidade nos pontos de avaliação do reservatório de Três Irmãos

\begin{tabular}{|c|c|c|c|c|c|c|c|c|c|c|c|c|c|c|c|c|c|c|c|c|c|}
\hline \multirow{2}{*}{$\begin{array}{l}\text { Ponto de } \\
\text { avaliação }\end{array}$} & \multirow{2}{*}{\multicolumn{2}{|c|}{$\begin{array}{c}\text { Coordenadas } \\
\text { UTM }\end{array}$}} & \multirow{2}{*}{$\begin{array}{l}\text { Área } \\
\left(\mathrm{m}^{2}\right)\end{array}$} & \multirow{2}{*}{\begin{tabular}{|c|} 
Nível de \\
infes- \\
tação
\end{tabular}} & \multicolumn{17}{|c|}{ Infestação de plantas aquáticas (\%) } \\
\hline & & & & & EGRNJ & EICCR & CERDE & NAJGU & POLHP & SAVMO & BRASU & EICAZ & SAVMI & POL & ELO & CYP & EGRDE & MYPBR & NYM & PISST & LUD \\
\hline 1 & 555409 & 7672318 & 500 & 3 & & 40 & & & 10 & & 40 & & 10 & & & & & & & & \\
\hline 2 & 556673 & 7671509 & 5.000 & 4 & 1 & 30 & 8 & 1 & 30 & & 20 & & 10 & & & & & & & & \\
\hline 3 & 560513 & 7671746 & 10.000 & 4 & 1 & 30 & 4 & & 20 & & 40 & & 5 & & & & & & & & \\
\hline 4 & 563373 & 7669121 & 2.000 & 4 & 0 & 45 & 0 & & 5 & 3 & 45 & & & & & 1 & & & & 1 & \\
\hline 5 & 567444 & 7670377 & 2.500 & 4 & 1 & 50 & 5 & 0 & 1 & & 40 & & 3 & & & & & & & & \\
\hline 6 & 568096 & 7669419 & 20.000 & 5 & 0 & 35 & 1 & & 60 & 4 & & & & & & & & & & & \\
\hline 7 & 569188 & 7669896 & 1.000 & 3 & 2 & 70 & & & & 3 & 25 & & & & & & & & & & \\
\hline 8 & 575448 & 7667951 & 10.000 & 4 & 0 & 18 & & & & & 80 & & 2 & & & & 0 & & & & \\
\hline 9 & 576588 & 7665065 & 8.000 & 4 & 5 & 10 & & & 15 & & 50 & 15 & 5 & & & & & & & & \\
\hline 10 & 579471 & 7664257 & 3.000 & 4 & & 10 & & 0 & 8 & 2 & & 80 & & & & & & & & & \\
\hline 11 & 582070 & 7663822 & 300 & 3 & & & & & & & & 100 & & & & & & & & & \\
\hline 12 & 581738 & 7664430 & & 1 & & & & & & & & & & & & & & & & & \\
\hline 13 & 577803 & 7665034 & 3.500 & 4 & 3 & 40 & & 2 & 10 & 4 & & 40 & & & & & & 1 & & & \\
\hline 14 & 575414 & 7669842 & 5.000 & 4 & 4 & 14 & & 1 & 40 & 1 & & 40 & & & & & & & & & \\
\hline 15 & 570206 & 7670663 & 5.000 & 4 & 2 & 10 & 2 & 1 & 45 & 30 & & 10 & & & & & & & & & \\
\hline 16 & 568079 & 7672255 & 1.000 & 3 & 1 & 50 & 8 & 1 & & & 35 & & & & & & & & & & 5 \\
\hline 17 & 569748 & 7675729 & 30.000 & 5 & 1 & 45 & 5 & 1 & & & & & 3 & & & 45 & & & & & \\
\hline 18 & 570963 & 7676863 & 40.000 & 5 & & 65 & 1 & 0 & & 2 & & 5 & & & & 25 & & & 1 & 1 & \\
\hline 19 & 564927 & 7673432 & 10.000 & 4 & 4 & 80 & 10 & 1 & & 5 & & & & & & & & & & & \\
\hline 20 & 561207 & 7675277 & 500 & 3 & 5 & 10 & 2 & 3 & & & & 80 & & & & & & & & & \\
\hline 21 & 561386 & 7672532 & 25.000 & 5 & & & 1 & 1 & & 3 & & 90 & & & & & & & 5 & & \\
\hline 22 & 559641 & 7678303 & 2.500 & 4 & 1 & 90 & 2 & 1 & & 5 & & & & & & & 1 & & & & \\
\hline 23 & 491155 & 7718482 & 500 & 3 & 35 & & & & & & & & & 25 & 40 & & & & & & \\
\hline 24 & 489349 & 7716483 & 1.500 & 4 & 40 & & & & & & & & & 30 & 30 & & & & & & \\
\hline 25 & 488754 & 7716409 & 500 & 3 & 50 & & & & & & & & & 30 & 20 & & & & & & \\
\hline 26 & 487464 & 7716711 & 2.000 & 4 & 90 & & & & & & & & & 10 & & & & & & & \\
\hline 27 & 487848 & 7718823 & 1.000 & 3 & 80 & & & & & & & & & 20 & & & & & & & \\
\hline 28 & 487458 & 7718861 & 5.000 & 4 & 60 & & & & & & & & & & 30 & & & 10 & & & \\
\hline 29 & 486890 & 7717800 & 1.500 & 4 & 80 & & & & & & & & & 20 & & & & & & & \\
\hline \multicolumn{5}{|c|}{$\mathrm{N}^{\mathrm{o}}$ de pontos com plantas da espécie } & 23 & 19 & 13 & 13 & 11 & 11 & 9 & 9 & 7 & 6 & 4 & 3 & 2 & 2 & 2 & 2 & 1 \\
\hline \multicolumn{5}{|c|}{$\mathrm{N}^{\mathrm{o}}$ de pontos com dominância } & 5 & 6 & 0 & 0 & 1 & 0 & 2 & 4 & 0 & 0 & 0 & 0 & 0 & 0 & 0 & 0 & 0 \\
\hline \multicolumn{5}{|c|}{ № de pontos com co-dominância } & 2 & 6 & 0 & 0 & 3 & 1 & 3 & 2 & 0 & 1 & 2 & 1 & 0 & 0 & 0 & 0 & 0 \\
\hline
\end{tabular}

Legenda: Nível de infestação: $1=0 \mathrm{~m}^{2} ; 2=1$ a $100 \mathrm{~m}^{2} ; 3=101$ a $1.000 \mathrm{~m}^{2} ; 4=1.001$ a $10.000 \mathrm{~m}^{2}$; e $5=>10.000 \mathrm{~m}^{2}$.

Espécie dominante: ocupa $50 \%$ ou mais da área infestada. Espécies co-dominantes: duas espécies com cobertura combinada de 50\% ou mais da área infestada. 
Tabela 5 - Composição da comunidade nos pontos de avaliação do reservatório de Paraibuna

\begin{tabular}{|c|c|c|c|c|c|c|c|c|c|c|c|c|c|c|c|c|}
\hline \multirow{2}{*}{$\begin{array}{c}\text { Ponto de } \\
\text { avaliação }\end{array}$} & \multirow{2}{*}{\multicolumn{2}{|c|}{$\begin{array}{c}\text { Coordenadas } \\
\text { UTM }\end{array}$}} & \multirow{2}{*}{$\begin{array}{l}\text { Área } \\
\left(\mathrm{m}^{2}\right)\end{array}$} & \multirow{2}{*}{$\begin{array}{c}\text { Nível de } \\
\text { infes- } \\
\text { tação }\end{array}$} & \multicolumn{12}{|c|}{ Infestação de plantas aquáticas (\%) } \\
\hline & & & & & POLLA & SAVMO & UTRGI & BRASU & EICCR & PANPU & POLHP & CYP & LUD & MYPBR & POLPL & PASRE \\
\hline 1 & 454767 & 7414119 & 50 & 2 & 30 & 40 & & 30 & & & & & & & & \\
\hline 2 & 455326 & 7414978 & 100 & 2 & 5 & 5 & 1 & 75 & & 10 & & & 2 & 2 & & \\
\hline 3 & 454160 & 7413996 & 100 & 2 & 10 & 10 & & 70 & & 10 & & & & & & \\
\hline 4 & 453825 & 7414569 & 5.000 & 4 & 90 & 3 & 1 & " 5 & "'nt & & & & & & & \\
\hline 5 & 453787 & 7414548 & 3.500 & 4 & 75 & 20 & 1 & & 4 & & & & & & & \\
\hline 6 & 453752 & 7414442 & 1.000 & 3 & 80 & 15 & 2 & & 3 & & & & & & & \\
\hline 7 & 453659 & 7414478 & 10.000 & 4 & 80 & 15 & 3 & & 2 & & & & & & & \\
\hline 8 & 453654 & 7414476 & 20.000 & 5 & 90 & 8 & 1 & & 1 & & & & & & & \\
\hline 9 & 453547 & 7414488 & 15.000 & 5 & 30 & 18 & & 50 & 2 & & & & & & & \\
\hline 10 & 452690 & 7415331 & 5.000 & 4 & 30 & 7 & 1 & 35 & 1 & 10 & 15 & 1 & & & & \\
\hline 11 & 452541 & 7415121 & 300 & 3 & 50 & & 3 & 15 & 2 & & 30 & & & & & \\
\hline 12 & 450741 & 7415911 & 20.000 & 5 & 80 & 15 & 5 & & & & & & & & & \\
\hline 13 & 4504496 & 7414443 & 100 & 2 & 40 & & & 50 & & 9 & & & & & & 1 \\
\hline 14 & 451913 & 7410240 & 300 & 3 & 60 & & 20 & & & 20 & & & & & & \\
\hline 15 & 454612 & 7412723 & 300 & 3 & & & & & & 20 & & & & & 80 & \\
\hline \multicolumn{5}{|c|}{$\mathrm{N}^{\mathrm{o}}$ de pontos com plantas da espécie } & 14 & 11 & 10 & 8 & 8 & 6 & 2 & 1 & 1 & 1 & 1 & 1 \\
\hline \multicolumn{5}{|c|}{$\mathrm{N}^{\mathrm{o}}$ de pontos com dominância } & 8 & 0 & 0 & 4 & 0 & 0 & 0 & 0 & 0 & 0 & 1 & 0 \\
\hline \multicolumn{5}{|c|}{$\mathrm{N}^{\mathrm{o}}$ de pontos com co-dominância } & 2 & 1 & 0 & 2 & 0 & 0 & 0 & 0 & 0 & 0 & 0 & 0 \\
\hline
\end{tabular}

Legenda: Nível de infestação: $1=0 \mathrm{~m}^{2} ; 2=1$ a $100 \mathrm{~m}^{2} ; 3=101$ a $1.000 \mathrm{~m}^{2} ; 4=1.001$ a $10.000 \mathrm{~m}^{2} ;$ e $5=>10.000 \mathrm{~m}^{2}$

Espécie dominante: ocupa $50 \%$ ou mais da área infestada. Espécies co-dominantes: duas espécies com cobertura combinada de 50\% ou mais da área infestada.

Tabela 6 - Composição da comunidade nos pontos de avaliação do reservatório de Jupiá

\begin{tabular}{|c|c|c|c|c|c|c|c|c|c|c|c|c|c|c|c|c|}
\hline \multirow{3}{*}{$\begin{array}{c}\begin{array}{c}\text { Ponto de } \\
\text { avaliação }\end{array} \\
1\end{array}$} & \multirow{2}{*}{\multicolumn{2}{|c|}{$\begin{array}{c}\text { Coordenadas } \\
\text { UTM }\end{array}$}} & \multirow{3}{*}{ 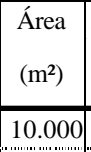 } & \multirow{3}{*}{\begin{tabular}{|c|}
$\begin{array}{c}\text { Nível de } \\
\text { infes- } \\
\text { tação }\end{array}$ \\
4 \\
4
\end{tabular}} & \multicolumn{12}{|c|}{ Infestação de plantas aquáticas (\%) } \\
\hline & & & & & \multirow{2}{*}{\begin{tabular}{|c|} 
EGRDE \\
15 \\
\end{tabular}} & \multirow{2}{*}{$\begin{array}{c}\text { EICCR } \\
10\end{array}$} & \multirow{2}{*}{$\frac{\text { TYP }}{25}$} & & \multirow{2}{*}{\begin{tabular}{|c|} 
EGRNJ \\
10
\end{tabular}} & \multirow{2}{*}{ SAVMO } & \multirow{2}{*}{\begin{tabular}{|c|} 
PISST \\
5
\end{tabular}} & \multirow{2}{*}{\begin{tabular}{|l|} 
EICAZ \\
\end{tabular}} & \multirow{2}{*}{\begin{tabular}{|c|} 
CERDE \\
25 \\
\end{tabular}} & \multirow{2}{*}{$\begin{array}{c}\text { BRASU } \\
10 \\
\end{array}$} & \multirow[t]{2}{*}{ POL } & \multirow[t]{2}{*}{ LUL } \\
\hline & 448396 & 7715781 & & & & & & & & & & & & & & \\
\hline 2 & 451395 & 7714946 & 15.000 & 5 & 20 & 5 & 42 & 10 & 5 & 1 & 1 & 5 & 10 & & 1 & \\
\hline 3 & 455313 & 7714214 & 10.000 & 4 & 3 & 5 & 85 & 1 & 1 & 2 & 2 & & 1 & & & \\
\hline 4 & 456801 & 7713287 & 20.000 & 5 & 30 & 15 & 30 & 5 & & & & 20 & & & & \\
\hline 5 & 459121 & 7712696 & 25.000 & 5 & 40 & 5 & 30 & 14 & 1 & & & 10 & & & & \\
\hline 6 & 460787 & 7712637 & 20.000 & 5 & 5 & 2 & 50 & 10 & 1 & 1 & 1 & 20 & 10 & & & \\
\hline 7 & 463465 & 7713416 & 20.000 & 5 & 10 & 5 & 40 & 10 & 1 & 3 & 1 & 15 & 15 & & & \\
\hline 8 & 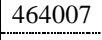 & 7713327 & 20.000 & 5 & 10 & 4 & 45 & 10 & 1 & 5 & & 5 & 20 & & & \\
\hline 9 & 464968 & 7713285 & 20.000 & 5 & 25 & 5 & 40 & 5 & 5 & 5 & & 5 & 10 & & & \\
\hline 10 & 464266 & 7712612 & 20.000 & 5 & 5 & 20 & 20 & 5 & & 5 & 5 & 25 & 5 & & 10 & \\
\hline 11 & 463481 & 7712698 & 10.000 & 4 & 10 & 25 & 25 & 18 & 1 & 5 & & 10 & & 1 & & 5 \\
\hline 12 & 459698 & 7711778 & 10.000 & 4 & 15 & 25 & 40 & 15 & 1 & 4 & 5 & 5 & & & & \\
\hline 13 & 453800 & 7713763 & 2.000 & 4 & 9 & 30 & 40 & 10 & 1 & 5 & 5 & & & & & \\
\hline 14 & 452867 & 7713625 & 10.000 & 4 & 5 & 10 & 50 & 20 & 1 & 5 & 5 & & 4 & & & \\
\hline 15 & 450719 & 7713671 & 5.000 & 4 & 5 & 40 & 40 & 10 & 1 & 2 & 2 & & & & & \\
\hline 16 & 448153 & 7713807 & 2.000 & 4 & 10 & 30 & 50 & 5 & & 3 & 2 & & & & & \\
\hline \multicolumn{5}{|c|}{ № de pontos com plantas da espécie } & 16 & 16 & 16 & 15 & 13 & 13 & 11 & 10 & 9 & 2 & 2 & 1 \\
\hline \multicolumn{5}{|c|}{$\mathrm{N}^{\mathrm{o}}$ de pontos com dominância } & 0 & 0 & 4 & 0 & 0 & 0 & 0 & 0 & 0 & 0 & 0 & 0 \\
\hline \multicolumn{5}{|c|}{$\mathrm{N}^{\mathrm{o}}$ de pontos com co-dominância } & 4 & 4 & 11 & 0 & 0 & 0 & 0 & 1 & 3 & 0 & 0 & 0 \\
\hline
\end{tabular}

Legenda: Nível de infestação: $1=0 \mathrm{~m}^{2} ; 2=1$ a $100 \mathrm{~m}^{2} ; 3=101$ a $1.000 \mathrm{~m}^{2} ; 4=1.001$ a $10.000 \mathrm{~m}^{2} ;$ e $5=>10.000 \mathrm{~m}^{2}$.

Espécie dominante: ocupa $50 \%$ ou mais da área infestada. Espécies co-dominantes: duas espécies com cobertura combinada de 50\% ou mais da área infestada. 
Tabela 7 - Composição da comunidade nos pontos de avaliação do reservatório de Jaguari

\begin{tabular}{|c|c|c|c|c|c|c|c|c|c|c|c|c|}
\hline \multirow{2}{*}{$\begin{array}{l}\text { Ponto de } \\
\text { avaliação }\end{array}$} & \multirow{2}{*}{\multicolumn{2}{|c|}{$\begin{array}{c}\text { Coordenadas } \\
\text { UTM }\end{array}$}} & \multirow{2}{*}{$\begin{array}{l}\text { Área } \\
\left(\mathrm{m}^{2}\right)\end{array}$} & \multirow{2}{*}{$\begin{array}{c}\text { Nível de } \\
\text { infes- } \\
\text { tação }\end{array}$} & \multicolumn{8}{|c|}{ Infestação de plantas aquáticas } \\
\hline & & & & & BRASU & SAVMO & POLLA & EICCR & PISST & CYP & MYPBR & TYP \\
\hline 1 & 378392 & 7429230 & 100 & 2 & 30 & & 70 & & & & & \\
\hline 2 & 376879 & 7426392 & 250 & 3 & 70 & 5 & 25 & & & & & \\
\hline 3 & 375008 & 7424252 & 750 & 3 & 5 & 15 & 80 & & & & & \\
\hline 4 & 373558 & 7423757 & 20.000 & "5 & 6 & 90 & 1 & 1 & 1 & 1 & & \\
\hline 5 & 372808 & 7423110 & 5.000 & 4 & 1 & 80 & 2 & 1 & 15 & 1 & & \\
\hline 6 & 372628 & 7423033 & 40.000 & 5 & 5 & 90 & 2 & 1 & 1 & 1 & & \\
\hline 7 & 372299 & 7422927 & 30.000 & 5 & & 95 & & 1 & 1 & 2 & 1 & \\
\hline 8 & 372481 & 7422921 & 30.000 & 5 & & 95 & & 1 & 1 & 2 & 1 & \\
\hline 9 & 373535 & 7423785 & 1.000 & 3 & 20 & 70 & 3 & 5 & 2 & & & \\
\hline 10 & 374716 & 7423303 & 200 & 3 & 10 & 70 & 4 & 5 & 1 & 10 & & \\
\hline 11 & 374653 & 7423273 & 50.000 & 5 & 40 & 10 & 10 & 5 & 5 & 30 & & \\
\hline 12 & 375217 & 7423384 & 5.000 & 4 & 25 & 30 & & 5 & 5 & 25 & & 10 \\
\hline 13 & 375678 & 7424345 & 200 & 3 & 50 & & 50 & & & & & \\
\hline 14 & 377301 & 7427595 & & 1 & & & & & & & & \\
\hline 15 & 382442 & 7430816 & & 1 & & & & & & & & \\
\hline \multicolumn{5}{|c|}{$\mathrm{N}^{\mathrm{o}}$ de pontos com plantas da espécie } & 11 & 11 & 10 & 9 & 9 & 8 & 2 & 1 \\
\hline \multicolumn{5}{|c|}{$\mathrm{N}^{0}$ de pontos com dominância } & 2 & 7 & 3 & 0 & 0 & 0 & 0 & 0 \\
\hline \multicolumn{5}{|c|}{$\mathrm{N}^{\mathrm{o}}$ de pontos com co-dominância } & 2 & 1 & 0 & 0 & 0 & 2 & 0 & 0 \\
\hline
\end{tabular}

Legenda: Nível de infestação: $1=0 \mathrm{~m}^{2} ; 2=1$ a $100 \mathrm{~m}^{2} ; 3=101$ a $1.000 \mathrm{~m}^{2} ; 4=1.001$ a $10.000 \mathrm{~m}^{2} ; 5=>10.000 \mathrm{~m}^{2}$.

Espécie dominante: ocupa $50 \%$ ou mais da área infestada. Espécies co-dominantes: duas espécies com cobertura combinada de $50 \%$ ou mais da área infestada.

Com base nos dados coletados, pode-se fazer as considerações que se seguem.

\section{Reservatório de Ilha Solteira}

Das seis espécies mais freqüentes, uma é submersa (Egeria najas), duas são flutuantes (Eichhornia crassipes e Salvinia molesta) e três são emersas (Eichhornia azurea, Polygonum spp. e Eleocharis spp.). Egeria najas ocorreu em 23 $(71,9 \%)$ pontos de avaliação, sendo dominante em 10 e co-dominante em 3, requerendo atenção nas próximas campanhas de monitorização, pois foi encontrada ao longo de todo o reservatório (rios Grande, Paraná e São José dos Dourados). Houve dominância (por uma espécie) em 20 pontos e co-dominância (por duas espécies) em 8. A área infestada média foi pequena $\left(2.439 \mathrm{~m}^{2}\right.$ por ponto de avaliação) e os níveis de infestação mais comuns foram 3 e 4 .

\section{Reservatório de Jaguari}

Das seis espécies mais freqüentes, três são emersas (Brachiaria arrecta, Polygonum lapathifolium e Cyperus spp.) e três são flutuantes (Salvinia molesta, Eichhornia crassipes e Pistia stratiotes). Salvinia molesta e Brachiaria arrecta ocorreram em $11(73,3 \%)$ pontos de avaliação, sendo a primeira dominante em 7 pontos e a segunda, em 2. Houve dominância em 11 pontos e co-dominância em 2. A área infestada média foi alta $\left(14.038 \mathrm{~m}^{2}\right.$ por ponto de avaliação) e os níveis de infestação mais comuns foram 3 e 5. Salvinia molesta ocupou grandes extensões do reservatório, principalmente próximo ao município de Santa Isabel, onde se observou que ela provoca dificuldades à pesca e à captação de água.

\section{Reservatório de Jupiá}

Das seis espécies mais freqüentes, duas são submersas (Egeria densa e E. najas), duas são flutuantes (Eichhornia crassipes e Salvinia molesta) e duas são emersas (Typha spp. e Cyperus spp.). Egeria densa, Eichhornia crassipes e Typha spp. ocorreram em todos os pontos de avaliação, mas apenas esta última chegou a estabelecer dominância (em 4 pontos). Houve dominância em 4 pontos e co-dominância em 11. Os níveis de infestação são altos: $50 \%$ dos pontos de avaliação tiveram nível 4 e $50 \%$, nível 5. A infestação média também foi elevada (13.688 $\mathrm{m}^{2}$ por ponto de avaliação), mesmo considerando que grande parte das plantas submersas não foi avaliada, por não terem atingido a superfície da água. 


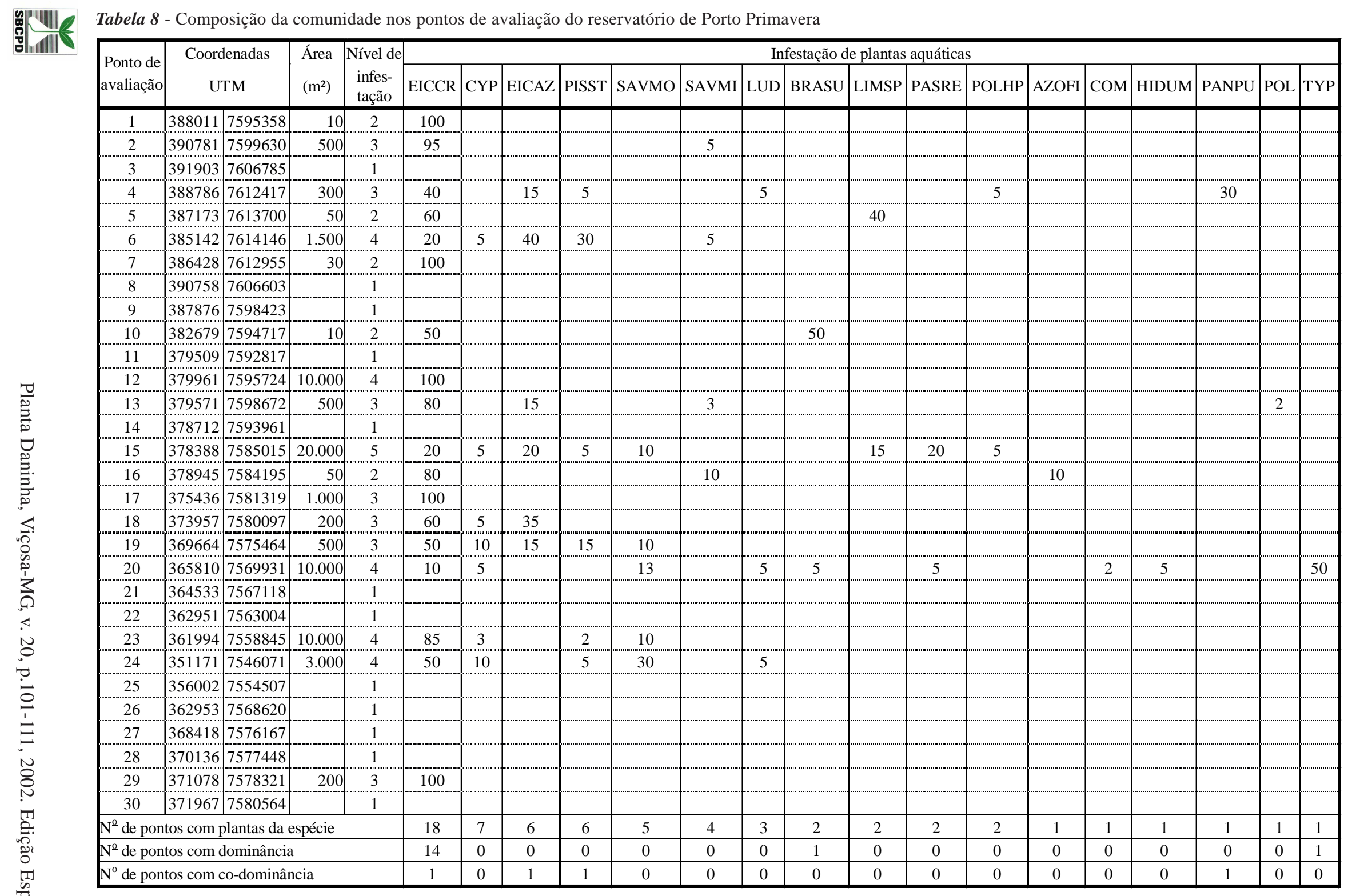

Legenda: Nível de infestação: $1=0 \mathrm{~m}^{2} ; 2=1$ a $100 \mathrm{~m}^{2} ; 3=101$ a $1.000 \mathrm{~m}^{2} ; 4=1.001$ a $10.000 \mathrm{~m}^{2}$; e $5=>10.000 \mathrm{~m}^{2}$.

Espécie dominante: ocupa 50\% ou mais da área infestada. Espécies co-dominantes: duas espécies com cobertura combinada de 50\% ou mais da área infestada. 
Tabela 9 - Resumo do levantamento de infestações de plantas aquáticas nos reservatórios da CESP

\begin{tabular}{|c|c|c|c|c|c|c|}
\hline \multirow[b]{2}{*}{ Avaliação } & \multicolumn{6}{|c|}{ Reservatório } \\
\hline & Ilha Solteira & Jaguari & Jupiá & Paraibuna & $\begin{array}{c}\text { Porto } \\
\text { Primavera }\end{array}$ & Três Irmãos \\
\hline $\mathrm{N}^{\mathbf{o}}$ de pontos de avaliação & 32 & 15 & 16 & 15 & 30 & 29 \\
\hline $\mathrm{N}^{\mathrm{o}}$ de pontos com plantas & $28(87,5 \%)$ & $13(86,7 \%)$ & $16(100 \%)$ & $15(100 \%)$ & $18(60 \%)$ & $28(96,6 \%)$ \\
\hline $\begin{array}{l}\text { Área infestada total }\left(\mathrm{m}^{2}\right) \text { nos } \\
\text { pontos de avaliação }\end{array}$ & 65.850 & 182.500 & 219.000 & 80.750 & 57.850 & 196.800 \\
\hline $\begin{array}{l}\text { Área infestada média }\left(\mathrm{m}^{2}\right) \text { nos } \\
\text { pontos de avaliação }\end{array}$ & 2.439 & 14.038 & 13.688 & 5.383 & 3.214 & 7.029 \\
\hline $\begin{array}{c}\text { Infestação ( } \text { № de pontos) }^{\text {Nível } 1} \\
\text { Nível } 2 \\
\text { Nível } 3 \\
\text { Nível } 4 \\
\text { Nível } 5\end{array}$ & $\begin{array}{c}4 \\
3 \\
12 \\
12 \\
1\end{array}$ & $\begin{array}{l}2 \\
1 \\
5 \\
2 \\
5\end{array}$ & $\begin{array}{l}0 \\
0 \\
0 \\
8 \\
8\end{array}$ & $\begin{array}{l}0 \\
4 \\
4 \\
4 \\
3\end{array}$ & $\begin{array}{c}12 \\
5 \\
7 \\
5 \\
1\end{array}$ & $\begin{array}{c}1 \\
0 \\
8 \\
16 \\
4\end{array}$ \\
\hline Espécies mais freqüentes & $\begin{array}{l}\text { EGRNJ } \\
\text { EICCR } \\
\text { EICAZ } \\
\text { POL } \\
\text { SAVMO } \\
\text { ELO }\end{array}$ & $\begin{array}{l}\text { BRASU } \\
\text { SAVMO } \\
\text { POLLA } \\
\text { EICCR } \\
\text { PISST } \\
\text { CYP }\end{array}$ & $\begin{array}{l}\text { EGRDE } \\
\text { EICCR } \\
\text { TYPLA } \\
\text { EGRNJ } \\
\text { SAVMO } \\
\text { PISST }\end{array}$ & $\begin{array}{c}\text { POLLA } \\
\text { SAVMO } \\
\text { UTRGI } \\
\text { BRASU } \\
\text { EICCR } \\
\text { PANPU }\end{array}$ & $\begin{array}{c}\text { EICCR } \\
\text { CYP } \\
\text { EICAZ } \\
\text { PISST } \\
\text { SAVMO } \\
\text { SAVMI }\end{array}$ & $\begin{array}{l}\text { EGRNJ } \\
\text { EICCR } \\
\text { CERDE } \\
\text { NAJGU } \\
\text { POLHP } \\
\text { SAVMO }\end{array}$ \\
\hline
\end{tabular}

\section{Reservatório de Paraibuna}

Das seis espécies mais freqüentes, três são emersas (Polygonum spp., Brachiaria arrecta e Brachiaria mutica), duas são flutuantes (Eichhornia crassipes e Salvinia molesta) e uma é submersa (Utricularia gibba). Polygonum spp. ocorreu em $14(93,3 \%)$ pontos de avaliação, sendo dominante em 8 e co-dominante em 2. Brachiaria arrecta ocorreu em 8 pontos e dominou em 4. As áreas mais infestadas encontramse distantes da UHE Paraibuna, e a ocupação é bastante restrita, pelo fato de o reservatório ser profundo. Houve dominância em 13 pontos e co-dominância em 2. A área infestada média foi de $5.383 \mathrm{~m}^{2}$ por ponto de avaliação e os níveis de infestação mais comuns foram 3, 4 e 5 .

\section{Reservatório de Porto Primavera}

Das seis espécies mais freqüentes, quatro são flutuantes (Eichhornia crassipes, Pistia stratiotes, Salvinia molesta e Salvinia mínima) e duas são emersas (Cyperus spp. e Eichhornia azurea). Eichhornia azurea ocorreu em 18 $(60,0 \%)$ pontos de avaliação, sendo dominante em 14 e co-dominante em 1. Houve dominância em 16 pontos e co-dominância em 2. A área infestada média foi de $3.214 \mathrm{~m}^{2}$ por ponto de avaliação e os níveis de infestação mais comuns foram 1 e 3 . A predominância de espécies flutuantes é em parte explicada pelo fato de o reservatório ser recente. As maiores infestações de plantas flutuantes ocorrem nos tributários e entre as árvores que não foram removidas antes da formação do reservatório. Nesses "paliteiros" observaram-se grandes áreas de Eichhornia crassipes desenvolvendo-se protegidas do fluxo d'água.

\section{Reservatório de Três Irmãos}

Das seis espécies mais freqüentes, três são submersas (Egeria najas, Ceratophyllum demersum e Najas guadalupensis), duas são flutuantes (Eichhornia crassipes e Salvinia molesta) e uma é emersa (Polygonum hydropiperoides). Eichhornia crassipes ocorreu em 19 (65,5\%) pontos de avaliação, sendo dominante em 6 e codominante em 6; Egeria najas ocorreu em 23 pontos $(79,3 \%)$ pontos, dominando em 5 e codominando em 2; outras espécies submersas, apesar de freqüentes, não dominaram nenhum ponto. Houve dominância em 18 pontos e co-dominância em 10. A área infestada média foi de $7.029 \mathrm{~m}^{2}$ por ponto de avaliação e os níveis de infestação mais comuns foram 3 e 4. Egeria najas, Egeria densa, Ceratophyllum demersum e 
Eichhornia crassipes merecem atenção nas próximas campanhas de monitorização.

\section{LITERATURA CITADA}

JENSEN, J. R.; DAVIS, B. A. Remote sensing of aquatic macrophyte distribution in selected South Carolina reservoirs. In: ASPRS-ACSM Annual Convention: Remote Sensing. Baltimore. Proceedings... Fall Church: ASPRSACSM, 1987. v. 1. p. 57-65.

MALTHUS, T. J.; GEORGE, D. G. Airborne remote sensing of macrophytes in Cefni Reservoir, Anglesey, UK. Aquatic Bot., v. 58, p. 317-332, 1997.
PEDRALLI, G. Macrófitos aquáticos, técnicas e métodos de estudos. Estudos de Biol., n. 26, p. 5-24, 1990.

SCHARDT, J. D. Florida aquatic plant survey report. Large Scale Hydrilla Management. Tallahassee: Florida Department of Environmental Protection. Bureau of Aquatic Plant Management, 1992. 83 p. (Technical Report, 942CGA).

SCHARDT, J. D.; LUDLOW, J. A. Florida aquatic plant survey report. Large Scale Hydrilla Management. Tallahassee: Florida Department of Environmental Protection. Bureau of Aquatic Plant Management, 1993. 63 p. (Technical Report, 952-CGA). 